\title{
Qual o valor da sedimentoscopia em urinas com características físico-quimicas normais?
}

\author{
Is it worth-while doing urine sediment microscopy in dipstick physicochemical normal samples?
}

\author{
José Aloysio da Costaval ${ }^{1}$ \\ Antônio de Pádua Massote ${ }^{2}$ \\ Cláudio Manoel Macedo Cerqueira ${ }^{3}$ \\ Adriane Pimenta da Costaval ${ }^{4}$ \\ Athos Auler ${ }^{5}$ \\ Gilberto José Martins ${ }^{6}$
}

\begin{tabular}{|c|c|}
\hline unitermos & resumo \\
\hline $\begin{array}{l}\text { Sedimentoscopia } \\
\text { Custos } \\
\text { Urinálise } \\
\text { Tira reagente }\end{array}$ & $\begin{array}{l}\text { O valor da sedimentoscopia em amostras de urina com exame físico-químico normal é } \\
\text { questionado na literatura médica estrangeira, sendo que muitos pesquisadores a julgam } \\
\text { desnecessária. Procurando contribuir para a elucidação desta dúvida e levando-se em conta } \\
\text { que a maioria dos laboratórios brasileiros sempre executa o exame completo, foi analisada } \\
\text { uma amostragem de uma clientela essencialmente ambulatorial, cujas requisições não } \\
\text { permitiam determinar se havia finalidade diagnóstica. Fez-se um estudo de } 10.234 \text { amostras, } \\
\text { que foram submetidas ao exame de rotina, sendo que } 5.000 \text { apresentaram exames físico- } \\
\text { químicos normais. Destas foi feita uma avaliação dos achados da microscopia do sedimento } \\
\text { que pudessem ser considerados possivelmente relevantes clinicamente. Entre esses achados, os } \\
\text { de maior incidência foram: cilindros ( } 29 \text { exames - 0,58\%) e piócitos em número igual ou } \\
\text { superior a cinco por campo ( } 26 \text { exames - 0,52\%). Todos os demais parâmetros foram } \\
\text { encontrados em números iguais ou inferiores a 0,4\%. Um percentual de } 98,02 \text { destas } \\
\text { amostras não revelou anormalidade à microscopia. A análise estatística pelo método do X² } \\
\text { ( } p<0,01 \text { ) sugere que não há necessidade da execução da sedimentoscopia nas urinas sem } \\
\text { anormalidades no exame físico-químico, o que representaria uma redução de } 48,85 \% \text { dos } \\
\text { sedimentos examinados. Desta forma se obteria uma sensível economia de tempo e de } \\
\text { gastos, sem nenhum prejuízo para o paciente. }\end{array}$ \\
\hline
\end{tabular}

abstract

The consequence of omitting urine sediment microscopy in dipstick physicochemical normal samples has been highly questioned in non Brazilian literature, in which many authors consider it not necessary. Considering that most of Brazilian laboratories always perform the complete urine examination and in order to clear this doubt, a study was done in 10.234 urine samples from essentially outpatients. Among these samples, 5000 showed normal dipstick physicochemical examination. In those, an evaluation of clinical possible relevant sediment microscopy elements was studied. Casts (29 samples - 0,58\%) and more than five leukocytes per field (26 samples -

$0,52 \%)$ showed to have the highest incidence, while all other sediment elements had their incidence numbers in $0,4 \%$ or less. No abnormal sediment elements were found in $98,02 \%$ of these samples. The $X^{2}$ method $(p<0,01)$ was used to perform the statistical analysis; the results showed that there is no need to do the urine sediment microscopy in dipstick

physicochemical normal samples. Based on these findings, it can be suggested that the suppression of the sediment examination in dipstick physicochemical normal samples will cause no impairment for both patient and physician. Then, significant savings of time and costs will be obtained, as 48,85\% sediment microscopy examination routinely performed in Brazilian laboratories could be safely suppressed.
1. Médico patologista clínico; ex-professor adjunto do Instituto de Ciências Biológicas da Universidade Federal de Minas Cerais; ex-chefe do laboratório central do Hospital das Clínicas da Universidade Federal de Minas Gerais; atual consultor do Laboratório São Marcos. 2. Médico patologista clínico do Laboratório São Marcos. 3. Médico patologista clínico do Laboratório São Marcos. 4. Médica veterinária; mestre; professora assistente da Escola de Veterinária da Universidade Federal de Minas Gerais. 5. Médico; estagiário do Laboratório São Marcos.

6. Farmacêutico bioquímico do Hospital Odilon Beherens e do Laboratório São Marcos. Estudo realizado no Laboratório São Marcos, Belo Horizonte. Trabalho apresentado no XXXII Congresso Brasileiro de Patologia Clínica e Medicina Laboratorial e no $X$ Congresso Mundial de Patologia Clínica. 


\section{Introducão}

O exame de urina de rotina, ou urinálise, compõe-se habitualmente de três etapas: o exame físico, o exame químico e a microscopia do sedimento.

Cada um deles tem seu valor, sendo os dois primeiros de execução mais simples e o último sendo considerado moderadamente complexo (10).

Em outros países, há muito se procurou simplificar o exame, abolindo-se a análise do sedimento sempre que o exame físico mostra uma urina límpida, de coloração normal e o exame químico, realizado com tira reagente, não revela anormalidade $(1,2,3,5,6$, $7,11,12,13,15)$. Tal norma foi adotada desde que respeitadas as características da clientela própria de cada serviço e as exigências da requisição médica $(10,15)$.

No Brasil, este procedimento praticamente não é adotado e a grande maioria dos laboratórios clínicos executa sempre as três etapas.

Considerando a possibilidade de economia a ser obtida em tempo e dinheiro que a supressão de uma sedimentoscopia, possivelmente desnecessária e que supostamente não resultaria em benefício para o cliente, foi proposto o presente trabalho, para, assim, avaliar a sua real necessidade. Caso ela seja comprovadamente irrelevante nas amostras de exame físico-químico normal, propõe-se a reavaliação da conduta de nossos laboratórios em executá-la compulsoriamente.

\section{Material e métodos}

Foram analisados retrospectivamente os resultados de 10.234 exames de urina de rotina realizados no Laboratório São Marcos, de Belo Horizonte, entre os meses de dezembro de 1998 e março de 1999.

As amostras procedentes da matriz, onde se localiza o setor técnico, eram deixadas em temperatura ambiente e examinadas no máximo em duas horas. As amostras procedentes das unidades de coleta eram colocadas em bolsas térmicas refrigeradas e enviadas para o setor técnico, onde também eram examinadas no tempo máximo de duas horas após a sua chegada.

A execução dos exames seguiu a padronização vigente no Laboratório São Marcos, semelhante à descrita na literatura (10), sendo todos submetidos ao exame físico (aspecto e coloração), à leitura da tira reagente de dez áreas (Combur $10 \mathrm{M}$, Roche) no aparelho Miditron $\mathrm{M}$ (Roche) e à microscopia do sedi- mento. A obtenção e a observação da microscopia obedeceram aos seguintes procedimentos:

- identificar a amostra e o tubo;

- colocar $12 \mathrm{ml}$ de urina em um tubo cônico;

- centrifugar entre 400 e $450 \mathrm{~g}$ por 5 minutos;

- ressuspender o sedimento em aproximadamente $0,5 \mathrm{ml}$ da própria urina ou soro fisiológico;

- colocar uma gota do sedimento no centro de uma lâmina;

- cobrir com lamínula de 22 × 22mm, inclinando-a gentilmente, de modo a evitar a formação de bolhas de ar;

- observar ao microscópio inicialmente com aumento de 100 vezes (objetiva de 10x). Correr praticamente toda a lâmina, observando a presença e distribuição dos diferentes elementos, principalmente dos de maior porte (cilindros, filamentos, grumos de piócitos etc.). Em seguida, focalizar com aumento de 400 vezes (objetiva de 40x) para se obter a média dos elementos presentes (piócitos, hemácias, células, cilindros, cristais etc.);

- transcrever os resultados obtidos para o mapa de trabalho.

Os elementos cuja presença ou quantificação foi analisada no trabalho são considerados referenciais para pessoas sadias dentro dos seguintes achados:

- piócitos isolados: média de 0 a 4 por campo;

- piócitos em grumos: ausentes;

- hemácias: média de 0 a 2 por campo;

- cilindros hialinos: raros ou ausentes por lâmina;

- flora microbiana: aparentemente normal;

- cristais anormais: ausentes;

- Trichomonas ou outros parasitos: ausentes;

- leveduras: ausentes ou raras em mulheres.

Os resultados dos exames, anotados em fichas individuais, foram separados em dois grupos, sendo o grupo I de resultados com quaisquer alterações no exame físico ou químico. O grupo II reunia as urinas sem anormalidades nestes exames.

Em seguida foram anotados os totais diários de cada grupo em planilhas próprias. As alterações observadas na microscopia do sedimento daqueles do grupo II foram registradas nas mesmas planilhas, adotados os seguintes valores referenciais mínimos:

- piócitos: za 5 por campo (400x);

- piócitos em grumos: qualquer achado por lâmina; 
- hemácias: $\geq$ a 3 por campo (400x);

- cilindros de quaisquer tipos: qualquer número por lâmina;

- flora microbiana: considerada aumentada em um critério que incluía:

- aparentemente normal (ausência de bactérias visíveis);

- discretamente aumentada (escassas bactérias visíveis);

- aumentada (visualização fácil de muitas bactérias);

- levedura ou pseudomicélios: qualquer achado por lâmina;

- Trichomonas sp. ou outros parasitos: qualquer achado por lâmina;

- cristais anormais: qualquer achado por lâmina.

O método do $X^{2}$ foi utilizado visando a avaliar a significância estatística dos achados patológicos do sedimento encontrados nas amostras com exame físico-químico normal. $\mathrm{O}$ valor preditivo negativo para cada elemento encontrado no sedimento, bem como do somatório destes elementos, foi calculado objetivando demonstrar que o exame físico-químico negativo pode, com segurança, representar a realidade, ou seja, a sedimentoscopia sem alterações dignas de nota.

\section{Resultados}

As amostras estudadas apresentaram a distribuição constante da Tabela 1, conforme a presença ou não de alterações físico-químicas.

Nas 5.000 amostras do grupo II, foram encontrados os seguintes elementos, adotados os parâmetros referenciais mínimos (Tabela 2 ).

Nas amostras em que se detectaram cilindros, 28 foram hialinos e um foi granuloso, não sendo encontrados Trichomonas, outros parasitos ou cristais anormais.

\section{Discussão e conclusões}

A preocupação com a importância da sedimentoscopia em amostras de urina que apresentem exa-

\section{Distribuição das amostras conforme presença ou não de alterações}

Tabela 1 físico-químicas

\begin{tabular}{clcc}
\hline Grupo & Características & Número absoluto & Número percentual \\
\hline II & $\begin{array}{l}\text { Urinas com alterações das } \\
\text { características físico-químicas }\end{array}$ & 5.234 & 51,15 \\
& $\begin{array}{l}\text { Urinas sem alterações das } \\
\text { características físico-químicas }\end{array}$ & 5.000 & 48,85 \\
\hline
\end{tabular}

\section{Elementos encontrados na sedimentoscopia de 5 mil urinas sem alteraçöes} Tabela 2 das características físico-químicas

\begin{tabular}{lcccc}
\hline Elementos & Número absoluto & Número percentual & Valor $X^{2}$ & Significância \\
Cilindros & 29 & 0,58 & 8,9 & $\mathrm{p}<0,01$ \\
Piócitos isolados & 26 & 0,52 & 11,6 & $\mathrm{p}<0,01$ \\
Grumos de piócitos & 07 & 0,14 & 37,35 & $\mathrm{p}<0,01$ \\
Hematúria & 07 & 0,14 & 37,35 & $\mathrm{p}<0,01$ \\
Flora aumentada & 07 & 0,14 & 37,35 & $\mathrm{p}<0,01$ \\
Leveduras ou pseudomicélios & 20 & 0,40 & 18,8 & $\mathrm{p}<0,01$ \\
\hline TOTAL & 96 & 1,92 & 20,0 & $\mathrm{p}<0,01$ \\
\hline
\end{tabular}


me físico-químico negativo não é recente na literatura estrangeira: a maioria dos estudos avaliados abordando este aspecto data de meados da década de 80 $(1-3,5,7-9,12,13)$. Os estudos preliminares foram realizados ainda na década de $70(6,11)$, e os trabaIhos mais recentes avaliam a aplicação dos resultados obtidos anteriormente em populações específicas, como, por exemplo, clínica emergencial pediátrica (4) ou adultos internados em clínica geral (2).

A percepção de que qualquer diminuição no custo operacional da urinálise levaria a grande economia, dada a sua freqüente realização, associada à visão crítica dos trabalhos estudados, levou à realização do estudo ora apresentado.

Nos diversos artigos científicos estudados, o número de amostras de urina avaliadas foi sempre inferior a 1.100. Para maior confiabilidade dos resultados e da análise estatística, optou-se por acumular 10.234 urinálises para a execução desse estudo (Tabela 1). Pelos motivos supracitados foi feita a escolha de população ambulatorial, como realizado em alguns estudos $(7-9,11,12)$.

Pela análise dos resultados obtidos, constata-se que apenas 1,92\% das amostras do grupo II apresentaram algum indício de anormalidade. Considerado isoladamente, nenhum dos elementos apresentou positividade superior a 0,6\% (Tabela 2).

Os resultados obtidos pelo método do $X^{2}$ demonstram que a possível discrepância encontrada entre os achados patológicos do sedimento em amostras com exame físico-químico normal não é estatisticamente significativa, mesmo considerando $p<0,01$ (Tabela 2).
Analisando-se conjuntamente os resultados obtidos com a tira reagente e os achados do exame físico (coloração e transparência), o valor preditivo negativo foi de $98 \%$ (Tabela 3), confirmando dados da literatura que o situam acima de $90 \%$ (5).

Embora métodos estatísticos diferentes tenham sido utilizados nos diversos estudos consultados, podese concluir que há grande nível de concordância entre os dados aqui apresentados e aqueles da literatura $(1,3,5,8)$.

Estes fatos nos permitem optar, inicialmente, pelo emprego seletivo da sedimentoscopia.

Como já discutido anteriormente, a clientela atendida pelo Laboratório São Marcos, no período em que se processou o trabalho, era essencialmente ambulatorial, e, pelas requisições dos exames, não se podia concluir se o exame possuía alguma finalidade diagnóstica ou se se tratavam de pedidos rotineiros.

No entanto, ao se decidir pela execução ou não da microscopia do sedimento, a procedência do exame ou a exigência manifesta do clínico devem ser consideradas. O National Committee for Clinical Laboratorial Standards (14), conforme citação de Ringsrud e Linné (10), orienta que "a decisão de se realizar a microscopia do sedimento deve ser de cada laboratório individualmente, com base na característica de sua clientela", e sugere que "ela deve ser feita quando solicitada pelo clínico, quando determinada pelo protocolo do laboratório ou quando se encontram anormalidades no exame físico-químico".

É muito enfatizado na literatura o custo que a sedimentoscopia acrescenta ao exame $(4,5,8,12)$.

Valor preditivo negativo para elementos encontrados na sedimentoscopia de Tabela 35 mil urinas sem alterações das características físico-químicas

\begin{tabular}{lcc} 
Elementos & Número absoluto & Valor preditivo negativo (\%) \\
Cilindros & 29 & 99 \\
Piócitos isolados & 26 & 99 \\
Grumos de piócitos & 07 & 99 \\
Hematúria & 07 & 99 \\
Flora aumentada & 07 & 99 \\
Leveduras ou pseudomicélios & 20 & 99 \\
\hline TOTAL & 96 & 98
\end{tabular}


De todos os trabalhos avaliados, somente um não observa redução significativa destes custos quando da supressão das sedimentoscopias desnecessárias. Nos Estados Unidos, adotada tal supressão, um trabalho mostra ser possível uma economia anual de US $\$ 8.352,00$ em um Serviço (1) e outro faz a projeção de US\$ 44 milhões de economia anual em todo o país (8).

Em nosso Serviço, no período em que foi feito o presente trabalho, a média diária de urinálises foi de 180. Destas, pelos critérios adotados, 88 sedimentoscopias se tornariam dispensáveis. Admitindo-se como sendo de dois minutos o tempo médio de cada exame do sedimento, haveria uma redução diária de aproximadamente três horas/técnico, ou seja, de $48,85 \%$ de seu tempo e custo. Considerando a variabilidade dos custos de realização da urinálise por região e que em nosso país a maioria dos laboratórios executa a sedimentoscopia em todas as amostras, a redução percentual de custos por técnico aqui proposta permite o cálculo adequado para cada situação ou laboratório em particular.

As finalidades deste trabalho são, pois, de provocar o debate e propor uma medida que seja econômica e que não traga prejuízo à qualidade do exame. Para tanto, visando a tornar mais fácil a seleção de quais amostras deveriam ser submetidas a sedimentoscopia, apresentamos um fluxograma de urinálise (Figura).

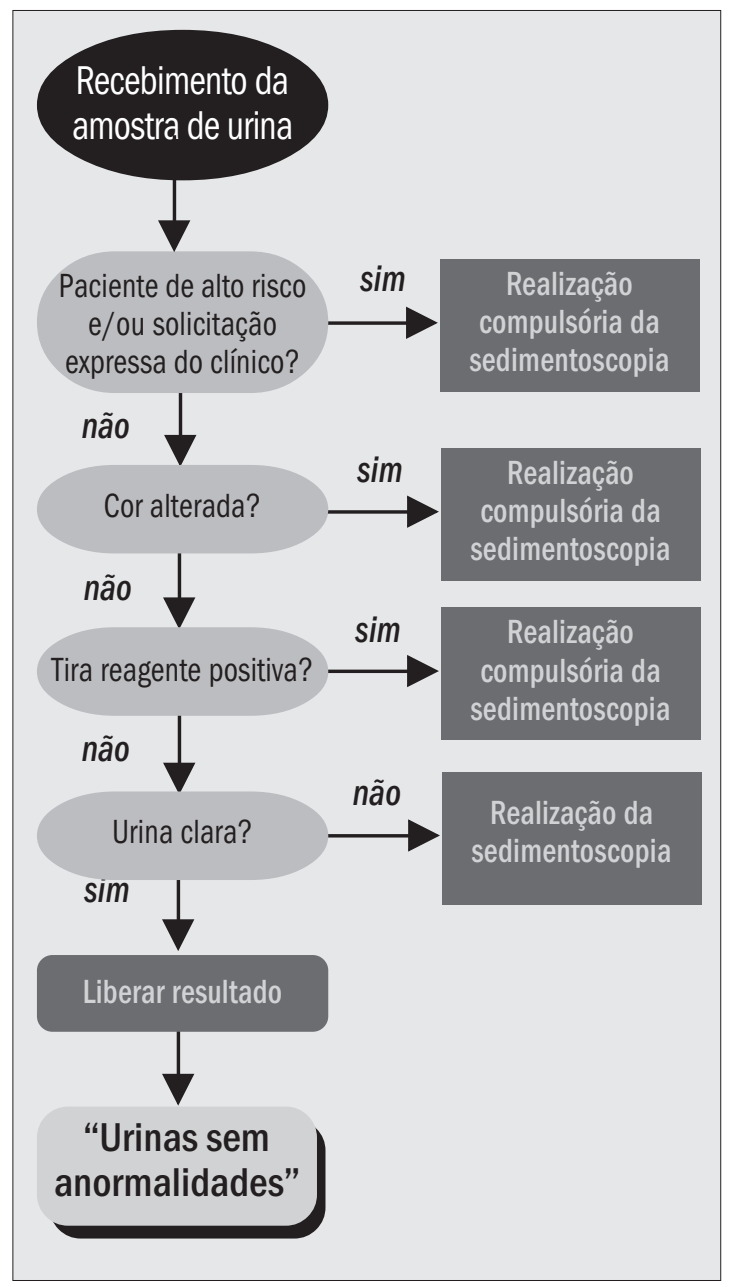

Figura - Fluxograma de urinálise. Adaptado de Christenson, R.H. et al.

\section{Referências}

1. Bartlett, R.C. \& Kaczmarczyk, L.A. Usefulness of microscopy examination in urinalysis A.J.C.P., 82(6): 713-5, 1984.

2. Benham, L. \& O'Kell, R.T. Urinalysis: minimizing microscopy (letter) Clin. Chem., 28: 1722, 1982.

3. Christenson, R.H.; Tucker, J.A.; Allen, E. Results of dipstick tests, visual inspection, microscopic examination of urine sediment and microbiological cultures of urine compared for simplifying urinalysis. Clin. Chem., 31: 448-51, 1985.

4. Craver, R.D. \& Abermanis, J.G. Dipstick only urinalysis screen for the pediatric emergency room. Pediatr Nephrol, 11: 331-3, 1997.

5. Hamoudi, A.S.; Bubis, S.C. \& Thompson, C. Can the cost of eliminating urine microscopy in biochemically negative urines be extended to the pediatric population? A.J.C.P., 86(5): 658-80, 1986.

6. Kutter, D. Testreifen zur Rationalisierung der Mikroskopischen Harnuntersuchung. Dtsch. Med. Wscht., 5(36): 1246-9, 1980.
7. Loo, S.Y.T. et al. Performance of a urine screening protocol. A.J.C.P., 85(4): 479-84, 1986.

8. Mariani, A.J. Disptick chemical urinalysis: an accurate cost effective screening test. J. Urol., 132: 64-6, 1984.

9. Morrison, M.C. \& Lum, G. Dipstick testing of urine-can it replace urine microscopy? A.J.C.P., 85(5): 590-4, 1986.

10. Ringsrud, K.M. \& Linné, J.J. Urinalysis and body fluids: a color text and atlas. St. Louis: Mosby, 1995.

11. Schumann, G.B. \& Greenberg, B.S. Usefulness of macroscopic urinalysis as a screening procedure: a preliminary report. A.J.C.P., 71(4): 452-6, 1979.

12. Shaw, S.T.; Poon, S.Y. \& Wong E.D. Routine urinalysis: is the dipstick enough? JAMA, 253: 1596-600, 1985.

13. Valenstein, P.N. \& Koepke, J.A. Unnecessary microscopy in routine urinalysis. A.J.C.P, 82(4): 444-8, 1986.

14. Villanova, PA. Routine urinalysis and collection, transportation and preservation of urine specimes, tentative guidelines. National Committee for Clinical Laboratory Standards, 12(26): 9, 1992.

15. Wenz, B. \& Lampasso, J.A. Eliminating unnecessary urine microscopy. A.J.C.P., 92(1): 78-81, 1989.
Endereço para correspondência José Aloysio da Costaval Laboratório São Marcos Avenida do Contorno, 9.797 - Prado CEP 30110-140 - Belo Horizonte-MC Fax: (31) 3275-4499 e-mail: dirtec@lbsmarcos.com.br 